\title{
TRANSFORM MODULATIONS FOR MOBILE COMMUNICATIONS
}

\author{
M.Lamarca, G. Vázquez \\ Dept. of Signal Theory and Communications, Polytecnhic University of Catalonia \\ c/Gran Capitán s/ $n$, 08034-Barcelona (SPAIN) \\ Tel: +34-3-4016436, Fax: +34-3-4016447, E-mail: \{xell,gregori\}@gps.tsc.upc.es
}

\begin{abstract}
In this paper a new modulation scheme called transform modulations is proposed which improves the system performance in frequency-flat fading mobile channels. They ameliorate the effect of the fadings over the transmitted signal by spreading the information in time, using a linear transform operator. The design of this schemes is discussed and its advantages ar shown by means of simulations. The relationship of this schemes with OFDM modulation is also analyzed.
\end{abstract}

\section{INTRODUCTION}

This work deals with block transmission strategies for mobile communications which are robust in front of frequency-flat fading $\left(\mathrm{F}^{3}\right)$ channels. In this paper some modulation schemes are proposed that achieve performance improvements without the need of increasing the transmission bandwidth or power, at the expense of increasing the constellation size. These techniques can be regarded as a generalization of the usual linear modulations (PSK, QAM, etc.) and they adopt the same policy as OFDM (Orthogonal Frequency Division Multiplexing) modulation.

The robustness of OFDM in front of $\mathrm{F}^{3}$-channels was first noticed in [1]. Afterwards, in [2], some techniques for multiplicative distortion compensation were proposed based on DFE (Decision Feedback Equalizer) approaches, whereas the channel response estimation was studied in [3] and a new blind equalization algorithm was proposed in [4].

In mobile communications subject to $\mathrm{F}^{3}$-channels, the performance improvement of OFDM on previously reported single-carrier schemes stems from the fact that in OFDM the symbols are transmitted in parallel. Thus, their duration is increased and, so, the probability of loosing one of them due to a fading decreases. OFDM modulation distributes the fading distortion among all symbols in a frame rather than concentrating it in a few of them, as happens in the single carrier case, and this allows for a decrease in the BER at the receiver. In some sense, the parallel transmission in OFDM provides robustness in front of the $F^{3}$-channel in the same way as diversity techniques do, even though it cannot be strictly considered as one of them because no redundancy is introduced at the transmitter or the receiver. According this point of view, this feature of OFDM will be named here temporal diversity.

In this paper it will be shown that many other transmission schemes share the same 'temporal diversity' of OFDM. The formulation of all these techniques is based on the transform modulations which are defined and analyzed in this paper. The design of the transform is also studied using perfor-

\footnotetext{
${ }^{0}$ This work has been partially supported by the Spanish Research Council (TIC-95-1022-C05-01, TIC-96-0500-C10-01) and by CIRIT/Generalitat de Catalunya (SGR0096)
}

mance and computational cost criteria. As shown in the next sections, the main conclusion from our analysis is that the Discrete Fourier Transform (DFT) and OFDM offer a good trade-off between performance and computational load.

During the development of this work, the same approach we propose here appeared in [5]. The basic transmission scheme applied there was the same one suggested in this paper. However, our conclusions are different from theirs, due to the fact that the two works have been developed under different constraints and design criteria. The differences betwreen the two analysis will arise in the next sections.

The paper is organized as follows. The transmission scheme is first introduced (section 2). Then the criteria applied for transform design is analyzed (section 3) and the transform is finally elected (section 4). Section 5 deals with the relationship between OFDM and the proposed transform modulation schemes. Finally, section 6 shows some results on the performance of the proposed schemes in $F^{3}$-channels.

\section{PROBLEM FORMULATION AND SIGNAL MODEL}

Figure 1 shows a block diagram of the proposed transmission scheme. The information symbols are rearranged into blocks of $N$ named $\underline{i}$ (serial-to-parallel conversion) and they are applied to a linear transform $I$, which is defined by an $(N \times N)$ matrix. The transformation output symbols make up a frame of the transmitted signal:

$$
\begin{aligned}
\underline{s}=\underline{T} \underline{i} & \underline{i}=\left[\begin{array}{llll}
i(n) & i(n+1) & \ldots i(n+N-1)
\end{array}\right]^{T} \\
\underline{s} & =\left[\begin{array}{llll}
s(n) & s(n+1) & \ldots s(n+N-1)
\end{array}\right]^{T}
\end{aligned}
$$

These symbols are applied to a modulator which transmits them according to a linear modulation:

$$
s(t)=\sum_{n} s(n) \cdot p(t-n T)
$$

being $p(t)$ the shaping pulse and $T$ the transmission rate. At the receiver, assuming the channel response is constant during one symbol transmission, the matched filter output is

$$
y(n)=s(n) \cdot c(n)+w(n)
$$

being $c(n)$ the $\mathrm{F}^{3}$-channel response and $w(n)$ an additivewhite Gaussian noise component with variance $\sigma_{w}^{2}$. Using matrix notation, this equation can be expressed as:

$$
\begin{gathered}
\underline{\underline{G}}=\operatorname{diag}\{c(n), c(n+1) \ldots c(n+N-1)\} \\
=\left[\begin{array}{cccc}
c(n) & 0 & \ldots & 0 \\
0 & c(n+1) & & \vdots \\
\vdots & & \ddots & \\
0 & \ldots & 0 & c(n+N-1)
\end{array}\right] \\
\underline{y}=\underline{\underline{C}} \underline{s}+\underline{w}
\end{gathered}
$$


The last step in the transmission process consists of the application of the inverse transform to the received sequence $\underline{y}$ in order to retrieve the information symbols $i(n)$ :

$$
\underline{r}=\underline{\underline{T}}^{-1} \underline{y}=\underline{\underline{T}}^{-1} \underline{\underline{C}} \underline{\underline{T}} \underline{i}+\underline{\underline{T}}^{-1} \underline{w}
$$

Equations (1)-(5) summarize the system under analysis. Notice that it can be considered as a generalization of the traditional linear modulations, for these can be expressed as a particular case of the proposed scheme with $\underline{\underline{T}}=\underline{\underline{I}}$.

When no transform is applied, $\underline{r}=\underline{C} \underline{i}+\underline{w}$ and the transmitted data appear at the receiver multiplied by the channel

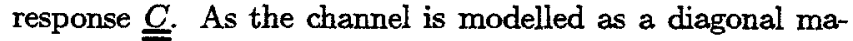
trix, no intersymbol interference (ISI) appears between the information symbols. However, if a deep fading occurs, it will cause the complete loss of the symbols which were being transmitted due to the temporary SNR degradation.

System behavior changes when a transform is employed. Then, if (1) is written in terms of the column vectors of matrix $\underline{T}$, the transmitted signal can be expressed as:

$$
\underline{\underline{T}}=\left[\underline{t}_{0} \underline{t}_{2} \ldots \underline{t}_{N-1}\right] \quad \Rightarrow \quad \underline{s}=\underline{\underline{T}} \underline{\underline{i}}=\sum_{k=0}^{N-1} \underline{t}_{k} i(n+k)
$$

In this equation it is evident that the $N$ information symbols are transmitted simultaneously, each one being associated to a different vector $\underline{t}_{k}$ and having duration $N T$.

In order to understand the performance of the system it is advisable to rephrase equation (1) as a basis change. The transformed symbols $s(n)$ can be understood as the projection of the information $\underline{i}$ over the row vectors of $\underline{T}$. Hence, the loss of one of the coefficients in $\underline{s}$ due to a fading is equivalent to the loss of the corresponding projection of $\underline{i}$ and, therefore, is equivalent to a partial degradation of all transmitted symbols. Thus, the transform achieves the desired temporal diversity. Since $\underline{T}^{-1} C T$ is not a diagonal matrix, the price which has to be paid for the temporal diversity is the appearance of ISI in $r$ and, so, the need of performing joint detection of all transmitted symbols.

Once it has been shown that the linear transform can provide the desired robustness in front of the $\mathrm{F}^{3}$-channel, next step is the design of the transform $T$. However, before moving on to it, it is worth mentioning that the transform modulations have at least two main drawbacks:

- The transmitted signal has no constant envelope because the modulation process increases the number of amplitude levels proportionally to its length $N$. This may impose severe constraints on the nonlinearity of the power amplifier stages.

- Symbol detection cannot be carried out until the whole frame has been received. This may introduce an important delay when the transform size $N$ is large.

\section{TRANSFORM DESIGN CRITERIA}

Transform selection must be based on its performance in front of the additive noise and the channel multiplicative distortion. Thus, the design criteria must take into account their contributions to the received signal degradation. The error in the received signal is:

$$
\underline{\varepsilon}=\underline{x}-\underline{i}
$$

Substituting (5) in the previous equation:

$$
\underline{\varepsilon}=\underline{d}_{i}+\underline{w}_{T} ; \quad \underline{d}_{i}=\left(\underline{\underline{T}}_{T}^{-1} \underline{\underline{C}} \underline{\underline{T}}-\underline{I}\right) \underline{\underline{i}}
$$

where $\underline{d}_{i}$ and $\underline{w}_{T}$ represent the ISI and noise contributions respectively. Therefore, if the channel is assumed deterministic, $\underline{i}$ is taken as a stochastic process realization and the usual uncorrelation hypothesis are made:

$$
E\left[\underline{i}^{H}\right]=\sigma_{i}^{2} \underline{\underline{I}} ; E\left[\underline{\underline{w}}^{H}\right]=\underline{\underline{0}}
$$

then the error $\underline{\varepsilon}$ covariance matrix is:

$$
E\left[\underline{\varepsilon} \underline{\varepsilon}^{H}\right]=\sigma_{i}^{2}\left(\underline{\underline{T}}^{-1} \underline{\underline{C}} \underline{\underline{T}}-\underline{\underline{I}}\right)\left(\underline{\underline{T}}^{-1} \underline{\underline{C}} \underline{\underline{T}}-\underline{\underline{I}}\right)^{H}+\sigma_{w}^{2} \underline{\underline{T}}^{-1}\left(\underline{\underline{T}}_{(10)}^{-1}\right)^{H}
$$

Given that $\underline{\varepsilon}$ generally is not Gaussian, its covariance matrix does not characterize it completely. However, it does allow for a first approximation to its behavior, as for large transform sizes $N$ the distribution function of $\underline{\varepsilon}$ is asymptotically Gaussian (central limit theorem).

Now, we can proceed to define the criteria under which the transform $\underline{\underline{T}}$ will be designed. The selected transform should fulfill four conditions:
1. Being a unitary matrix
2. Providing the desired temporal diversity
3. To ease the process of channel distortion com- pensation
4. Having a low computational cost

Next, this four conditions are analyzed.

\subsection{Unitarity}

The transform matrix must be unitary $\left(\underline{\underline{T}} \underline{T}^{H}=\underline{T}^{H} T=\underline{\underline{T}}\right)$ in order to preserve the Euclidean distance properties and correlation properties of the information symbols and the additive noise term.

When this condition is fulfilled, if the diagonal matrix $\underline{\underline{D}}$ is defined as:

$$
\underline{\underline{D}}=\underline{\underline{C}}^{H}-\underline{\underline{C}}^{H}-\underline{\underline{C}}+\underline{\underline{I}}
$$

then the channel distortion error covariance matrix (see (8)) and the variance due to channel distortion can be written as:

$$
\begin{aligned}
& E\left[\underline{d}_{i} \underline{\underline{d}}_{i}^{H}\right]=\sigma_{i}^{2}\left(\underline{\underline{T}}^{H} \underline{\underline{C}} \underline{\underline{T}}-\underline{\underline{I}}\right)\left(\underline{\underline{T}}^{H} \underline{\underline{C}} \underline{\underline{T}}-\underline{\underline{I}}\right)^{H} \\
& =\sigma_{i}^{2} \underline{\underline{T}}^{H}\left(\underline{\underline{C} \underline{C}^{H}}-\underline{\underline{C}}^{H}-\underline{\underline{C}}+\underline{\underline{I}}\right) \underline{\underline{T}}=\sigma_{i}^{2} \underline{\underline{T}}^{H} \underline{\underline{D}} \underline{\underline{T}} \\
& E\left[\underline{d}_{i}^{H} \underline{d}_{i}\right]=\operatorname{tr}\left\{E\left[\underline{d}_{i} \underline{d}_{i}^{H}\right]\right\}=\sigma_{i}^{2} \cdot \operatorname{tr}\left\{\underline{\underline{T}}^{H} \underline{\underline{D}} \underline{\underline{T}}\right\} \\
& =\sigma_{i}^{2} \cdot \operatorname{tr}\left\{\underline{\underline{D}} \underline{\underline{T}} \underline{\underline{T}}^{H}\right\}=\sigma_{i}^{2} \cdot \operatorname{tr}\{\underline{\underline{D}}\}
\end{aligned}
$$

being $\operatorname{tr}\{\cdot\}$ the trace operator. Therefore, the total distortion introduced by the channel does not depend on the selected transform. What makes the difference between the distinct unitary $\underline{\underline{T}}$ is its distribution between all components in $\underline{d}_{i}$.

\subsection{Temporal diversity}

The transform $\underline{\underline{T}}$ must provide the desired temporal diversity, that is, the channel distortion must be shared equally by all the transmitted symbols, no matter what is the multiplicative channel realization. Mathematically, the temporal diversity can be expressed as all the components in the error signal $\underline{\varepsilon}$ having the same variance:

$$
\text { main diagonal }\left\{E\left[\underline{d}_{i} \underline{d}_{i}^{H}\right]\right\}=\text { constant }
$$

where the expectation operator is performed with respect to the transmitted signal. When using a unitary matrix, this condition can be further developed to get to more useful results. Equation. (6) can be expressed in terms of the column vectors of $\underline{\underline{T}}$ as: 


$$
E\left[\underline{d}_{i} \underline{d}_{i}^{H}\right]=\sigma_{i}^{2} \underline{\underline{T}}^{H} \underline{\underline{D}} \underline{\underline{T}}=\left[\begin{array}{l}
\underline{\underline{t}}_{0}^{H} \\
\underline{\underline{t}}_{1}^{H} \\
\vdots \\
\underline{t}_{N-1}^{H}
\end{array}\right] \underline{\underline{D}}\left[\begin{array}{lll}
\underline{t}_{0} & \underline{t}_{1} & \ldots \\
\underline{t}_{N-1}
\end{array}\right]
$$

Therefore, the main diagonal coefficients in the covariance matrix are:

$$
\text { main diagonal }\left\{E\left[\underline{d}_{i} \underline{d}_{i}^{H}\right]\right\}=\left\{\underline{t}_{k}^{H} \underline{\underline{D}} \underline{t}_{k}\right\}_{0 \leq k<N-1}
$$

As the matrix $\underline{\underline{D}}$ is diagonal, the only way to verify equation (15) for any multiplicative channel realization is to force that:

$$
\left|\underline{t}_{1}\right|=\left|\underline{t}_{2}\right|=\ldots=\left|\underline{t}_{N}\right|
$$

where $|\cdot|$ stands for the absolute value operator. Notice the relationship between this result and the ones obtained in [5], although there only the real transform case was analyzed.

\subsection{Channel distortion compensation}

The introduction of the transform cannot hinder either the receiver capability of optimally detecting the information symbols or that one of easily describing and understanding the effect of channel distortion over the transmitted signal. In this context, in [5] it was said that the optimum receiver was too complex to be carried out, so that in practical applications the Minimum Mean Squared Error linear receiver (MMSE) had to be employed. This section is intended to show that there is a case where this optimum receiver is computationally feasible. That is possible because the multiplicative distortion can be analyzed in a different framework where the matrix notation is replaced by that one of convolution and aliasing. Let's assume the transform $T$ corresponds to a scaled transpose Vandermonde matrix:

$$
\underline{\underline{T}}=\alpha\left[\begin{array}{llll}
1 & \rho_{0} & \cdots & \rho_{0}^{N-1} \\
1 & \rho_{1} & & \rho_{1}^{N-1} \\
\vdots & & \ddots & \vdots \\
1 & \rho_{N-1} & \cdots & \rho_{N-1}^{N-1}
\end{array}\right]
$$

and let's describe the input sequence by its associated polynomial (or equivalently its z-transform):

$$
\mathbf{i}(x)=i(n)+i(n+1) x+\ldots+i(n+N-1) x^{N-1}
$$

Then, the transformation process can be understood as the computation of the residue $e^{1}$ of $i(x)$ modulus the first order polynomials which have roots $\rho_{0} \ldots \rho_{N-1}$ :

$$
\begin{aligned}
& s(n+k)=\left.\alpha \cdot \mathbf{i}(x)\right|_{x=\rho_{k}} \quad 0 \leq k<N-1 \\
& s(n+k)=\alpha \cdot \mathbf{i}(x) \quad\left(\bmod \left(x-\rho_{k}\right)\right)
\end{aligned}
$$

Besides, if the $N$-degree polynomials $\mathbf{c}(x), \mathbf{r}(x)$ and $\mathbf{w}(x)$ are defined as those associated to the channel, received signal and noise so that they verify that

$$
\begin{aligned}
& \alpha \cdot c(n+k)=\mathbf{c}(x)\left(\bmod \left(x-\rho_{k}\right)\right) \quad 0 \leq k<N-1 \\
& y(n+k)=\mathbf{r}(x) \quad\left(\bmod \left(x-\rho_{k}\right)\right) \quad 0 \leq k<N-1 \\
& w(n+k)=\mathrm{w}(x)\left(\bmod \left(x-\rho_{k}\right)\right) \quad 0 \leq k<N-1
\end{aligned}
$$

then it follows that equation (5) can be rephrased as:

$$
\mathbf{r}(x)=\mathbf{c}(x) \cdot \mathbf{i}(x)+\mathbf{w}(x) \quad\left(\bmod \prod_{0 \leq k<N-1}\left(x-\rho_{k}\right)\right)
$$

\footnotetext{
${ }^{1}$ For polynomial residue and modular algebra properties see [6]
}

Thus, the channel distortion can be considered as the combination of a linear convolution (polynomial product) and a weighted aliasing (polynomial residue) operation. Equation (21) shows the advantages of Vandermonde matrices. First, convolution and aliasing are very well known operations in signal processing, so understanding the channel distortion in the transformed domain is simplified. Second, in many cases equation (21) is easier to deal with than (5), especially when the channel response $c(n)$ fits a polynomial $c(x)$ of degree much lower than $N$, as is the case of the slowly varying mobile channels when the DFT is applied.

\subsection{Computational load}

The transform computational cost may be important, especially when the transform size $N$ is large. It would be desirable to employ a transform which can be implemented by means of fast efficient algorithms.

\section{TRANSFORM ELECTION}

Once the design criteria have been defined they must be applied to choose the transform which will be employed to achieve the desired temporal diversity. Only unitary transforms will be considered, for if this condition was not verified it would preclude proper system operation.

With respect to the temporal diversity criterion, there is an unlimited number of unitary transforms which verify (17), e.g. the Discrete Fourier Transform (DFT), the Walsh-Hadamard Transform (WHT) and the circulant matrices associated to CAZAC (Constant-Amplitude ZeroAutocorrelation) sequences.

The Fourier matrix:

$$
\underline{=}=\frac{1}{\sqrt{N}}\left[\begin{array}{cccc}
1 & 1 & \cdots & 1 \\
1 & e^{-j \frac{2 \pi}{N}} & & e^{-j \frac{2 \pi}{N}(N-1)} \\
\vdots & & \ddots & \vdots \\
1 & e^{-j \frac{2 \pi}{N}(N-1)} & & e^{-j \frac{2 \pi}{N}(N-1)^{2}}
\end{array}\right]
$$

satisfies equation (17). Besides, in this case the temporal diversity is evident. If $\underline{\underline{T}}=\underline{\underline{E}}$ it follows that matrix $\underline{\underline{T}}^{H} \underline{\underline{ }} \underline{\underline{T}}$ in equation (5) is a circulant matrix. Therefore, the channel distortion can be regarded as a circular convolution of $i(n)$ and $c(n)$, and so it is clear that it introduces a constant variance (ISI) in all transmitted symbols.

The Walsh-Hadamard Transform (WHT) ${ }^{2}$ also verifies the temporal diversity condition, since all its coefficients take values \pm 1 . In this case, the temporal diversity is also evident because when this transform is applied the matrix $\underline{\underline{T}}^{T} \underline{\underline{ }} \underline{\underline{T}}$ performs a dyadic convolution ([7]).

Finally, as the last example, the circulant matrices defined based on the circular convolution by CAZAC sequences:

$$
\begin{aligned}
& \left\{q_{n}\right\}_{0 \leq n \leq N-1}: \sum_{n=0}^{N-1} q_{(n+k) \bmod N} \cdot q_{n}^{*}=\delta(n-k) \\
& T=\operatorname{circ}\left\{q_{n}\right\}=\left[\begin{array}{llll}
q_{0} & q_{1} & \cdots & q_{N-1} \\
q_{N-1} & q_{0} & & q_{1} \\
\vdots & & \ddots & \vdots \\
q_{1} & q_{2} & \cdots & q_{0}
\end{array}\right]
\end{aligned}
$$

also satisfy the temporal diversity property and they are unitary. However, their application to the multiplicative channel has no straightforward interpretation.

The third condition imposed on the transform matrix (ease of interpretation and compensation of the channel distortion) is the most selective one, for most known transforms

\footnotetext{
${ }^{2}$ Notice that transform modulations can be understood as a generalization of the time $(\underline{\underline{T}}=\underline{\underline{I}})$, frequency $(\underline{\underline{T}}=\underline{\underline{F}})$ and code (e.g WHT) division multiplexing schemes
} 
do not correspond to Vandermonde matrices. In fact, it can be demonstrated that, if the unitarity and temporal diversity conditions are imposed on the Vandermonde matrix in (18) at the same time, the only ones which verify them are those which can be expressed as

$$
\underline{\underline{T}}=\epsilon^{j \theta} \underline{\underline{P}} \underline{\underline{F}} \underline{\underline{\Phi}}
$$

where $\theta$ is an arbitrary phase, $\underline{P}$ is a permutation matrix, $\underline{E}$ is the Fourier matrix in (22) and $\Phi$ introduces an arbitrary linear phase in the Fourier matrix rows:

$$
\Phi=\left[\begin{array}{llll}
1 & 0 & \cdots & 0 \\
0 & e^{j \varphi} & & \vdots \\
\vdots & & \ddots & 0 \\
0 & \cdots & 0 & e^{j(N-1) \varphi}
\end{array}\right]
$$

From the computational point of view, in the literature fast algorithms have been published for many unitary transforms. Among those transforms which verify equation (17), both DFT and WHT are appropriate, since methods for computing it in $N \log _{2} N$ operations have been described for both of them. However, the WHT has the additional advantage of being a linear transform which requires no multiplications for its implementation.

In conclusion, the DFT and its associated alternatives according to equation (23) constitute the only transforms which satisfy the desired conditions described in section 3 . The WHT will be a good choice for implementing temporal diversity in those cases where algorithm simplicity is considered to be more important than system performance.

Both the DFT and WHT provide the same temporal diversity, but the differences arise when dealing with channel distortion compensation. If the DFT is applied, the channel estimation can be integrated as part of the data frame ([3]) and both linear equalization (MMSE, zero forcing,..., see section 6) and non-linear (DFE equalization algorithms ([2]), etc.) can be applied, as well as optimum detection techniques (see section 6) which significantly improve system performance. On the contrary, if the WHT is applied, only the linear equalization can be easily employed.

\section{EQUTVALENCE OF DFT MODULATION AND OFDM}

The transform modulations proposed in section II are based on a single-carrier transmission. Therefore, they cannot be equivalent to OFDM. However, when the elected transform is the DFT, the equivalent discrete-time system at 1 sam$\mathrm{ple} / \mathrm{symbol}$ coincides with that one of OFDM discretized at the same sampling rate (see [8] for the description of OFDM in terms of the DFT). Hence, since there is no difference in the analysis and performance of both systerns when working at 1 sample/symbol, the results obtained for OFDM in the past can be applied directly to the transform modulation scheme. Nevertheless, differences appear when working with the analog signal or when that one is oversampled:

- the transmitted signal spectrum will be different in both cases. In the transform modulations it will depend on the shaping pulse $p(t)$.

- in the case of the transform modulations the transmitted signal is cyclostationary with period $T$, whereas the OFDM modulated signal is not.

According to the results in the previous sections and the mentioned equivalence, it follows that the original application of OFDM to the $F^{3}$-channel was very fortunate, even though was not deliberate because no other choice was considered. In our opinion, either the DFT-based transform modulation or OFDM should be the transmission schemes to be applied if the temporal diversity is to be achieved.

\section{SIMULATIONS}

In this section, some results are shown on the performance improvement achieved by the transform modulations techniques in a $\mathrm{F}^{3}$-channel.

Figures 2 and 3 show the BER for the transmission of a BPSK signal over a Rayleigh fading channel with normalized Doppler $f_{d} T=0.0037$, corresponding to a $25 \mathrm{~Kb} / \mathrm{s}$ transmission at $1 \mathrm{GHz}$ in a vehicle moving at $100 \mathrm{Km} / \mathrm{h}$.

Figure 2 displays the results obtained for the case of no temporal diversity (NTD) and the case of applying a DFT of size $N=8,32,128,512,1024$. In the diversity-less case, the data estimation has been carried out assuming the channel phase evolution was known. In the transform modulation case, the channel is assumed to be completely known and channel distortion is linearly compensated by means of a MMSE equalizer, that is, the signal is equalized before the inverse transform is applied according to:

$$
\underline{\hat{\imath}}=\underline{\underline{T}}^{-1} \underline{\underline{E}} \underline{\underline{y}}
$$

being $\underline{\underline{T}}^{-1}$ the DFT matrix and $\underline{\underline{E}}$ the diagonal equalization matrix

$$
\underline{\underline{E}}=\operatorname{diag}\{e(n), e(n+1) \ldots e(n+N-1)\}
$$

with coefficients ${ }^{3}$ :

$$
e(n)=\frac{c^{*}(n)}{\sigma_{w}^{2}+|c(n)|^{2}}
$$

Notice that the BER improvement is significant even for small transform sizes.

Figure 3 shows the different performance obtained when a linear MMSE equalizer and an optimum detection algorithm are applied. Here, a DFT of size is $N=128$ has been employed. The MMSE plot is the same one as in figure 2. The optimum detection has been performed assuming the channel response was known and it had been approximated in the transformed domain by its 5 lower frequency coefficients (elements $\# 0,1,2,126,127$ of the DFT of $c(n))$. Since the multiplicative channel behaves in the frequency domain as a circular convolution ([3]), the Maximum Likelihood estimate of the transmitted symbols has been obtained by adapting the MLSE (Maximum Likelihood Sequence Estimation) based on the Viterbi algorithm ([10]) to the circular convolution case. Figure 4 shows the modulus of a realization of the Rayleigh channel and its DFT to evidence that the 128-coefficient $c(n)$ can be well approximated by the low frequency components.

Figure 5 compares the performance of the DFT and WHT for a QPSK signal in the same scenario as before and using the MMSE equalizer of eq. (25)-(27). Both provide approximately the same BER, for the differences between the two plots can be due to the limited number of runs averaged in the figure. Notice however that if the WHT were employed, the maximum likelihood detector would only be possible at the expense of a much higher computational cost.

\section{CONCLUSIONS}

A new linear modulation scheme has been presented which improves the performance in $F^{3}$-channels. The proposed modulation is based on the application of a linear transform. The transform design has been analyzed. It has been shown that the DFT and WHT have the desired properties of unitarity, temporal diversity and moderate computational cost,

\footnotetext{
${ }^{3}$ The MMSE linear equalizer looks alike that one of the filter for a frequency selective channel (see [9, Pag.337]). This equation appeared also in [5]
} 
but the optimum receiver complexity is much lower in the DFT than in WHT. Finally, the relationship of these schemes with OFDM has been studied too.

\section{REFERENCES}

[1] L.J.Cimini, 'Analysis and simulation of a digital mobile channel using Orthogonal Frequency Division Multiplexing', IEEE Trans. on Communications, Vol. COMM-33, pp. 665-675, July 1985

[2] J.Ahn, H.S.Lee, 'Frequency domain equalisation of OFDM signals over frequency nonselective Rayleigh fading channels', Electronic Letters, Vol.29, No.16, pp.14761477, August 1993

[3] M.Lamarca, G.Vázquez, 'Channel estimation for transform modulations in mobile communications', Proc. of Eighth European Signal Processing Conference (EUSIPCO’96), Trieste (Italy), pp.1977-1980, September 1996

[4] M.Lamarca,G.Vázquez, 'Multichannel Receivers for OFDM and TDMA in mobile communications', Proc. of Intl. Conf. on Acoustics, Speech and Signal Processing, Munich(Germany), pp.3865-3868, April 1997

[5] D.Rainish, 'Diversity transform for fading channels', IEEE Trans. on Communications, Vol.44, No.12, pp.1653-1661, December 1996

[6] R.E.Blahut, Fast algorithms for digital signal processing, Addison-Wesley 1985

[7] N.Ahmed, K.R.Rao, Orthogonal transforms for digital signal processing, Springer-Verlag, 1975

[8] S.P.Weinstein, P.M.Ebert, 'Data transmission by Frequency Division Multiplexing using the Discrete Fourier Transform', IEEE Trans. on Communication Technology, Vol. COM-19, No.5, pp.628-634, October 1971

[9] S.Benedetto, E.Biglieri, V.Castellani, Digital Transmission Theory, Prentice-hall, 1987

[10] G.D.Forney, 'Maximum-Likelihood Sequence Estimation of digital sequences in the presence of intersymbol interference', IEEE Trans. on Information Theory, Vol.IT-18, pp.363-378, May 1972

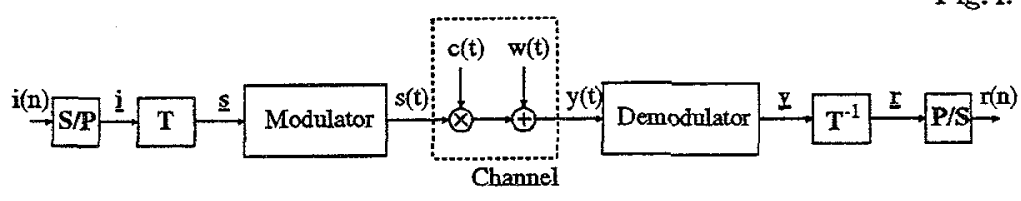

Fig.1. Block diagram of the proposed transmission system

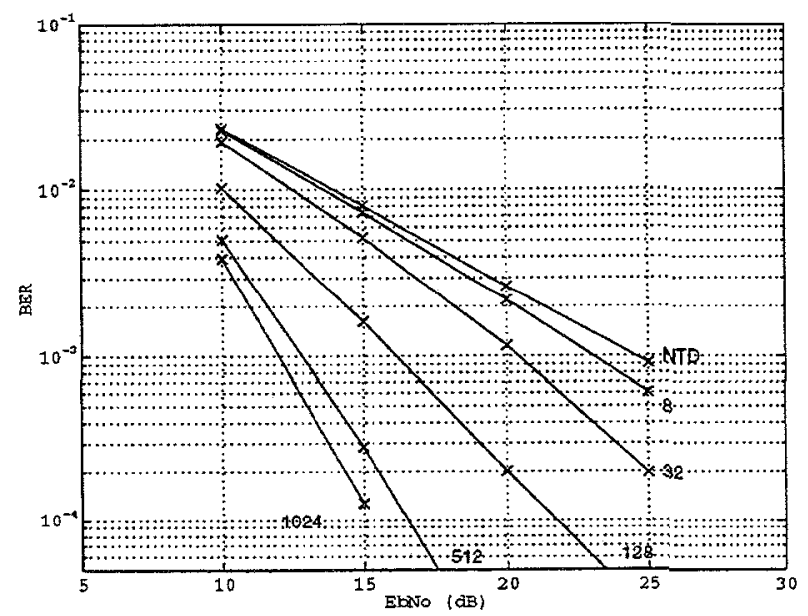

Fig.2. BER for the transmission without temporal diversity (NTD) and for the transform modulation scheme.

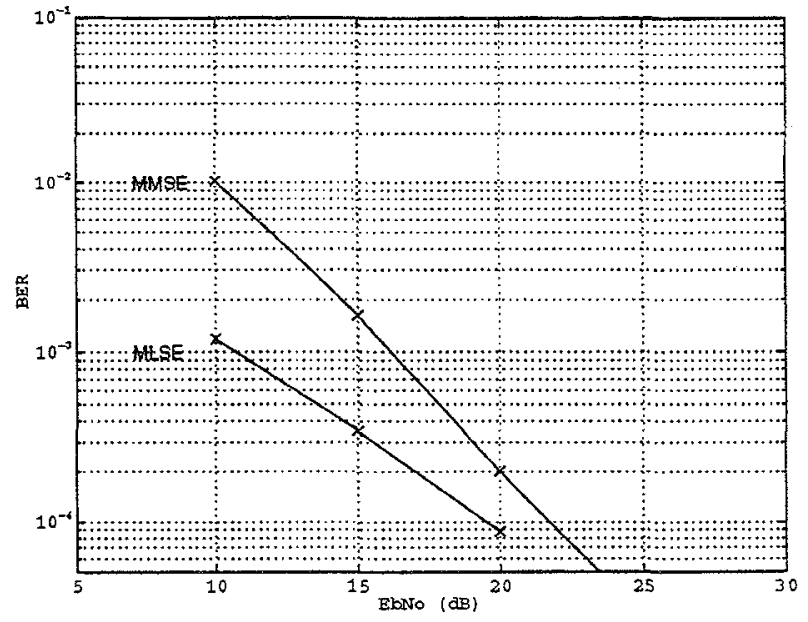

Fig.3. MMSE linear equalization vs. Maximum Likelihood estimation.
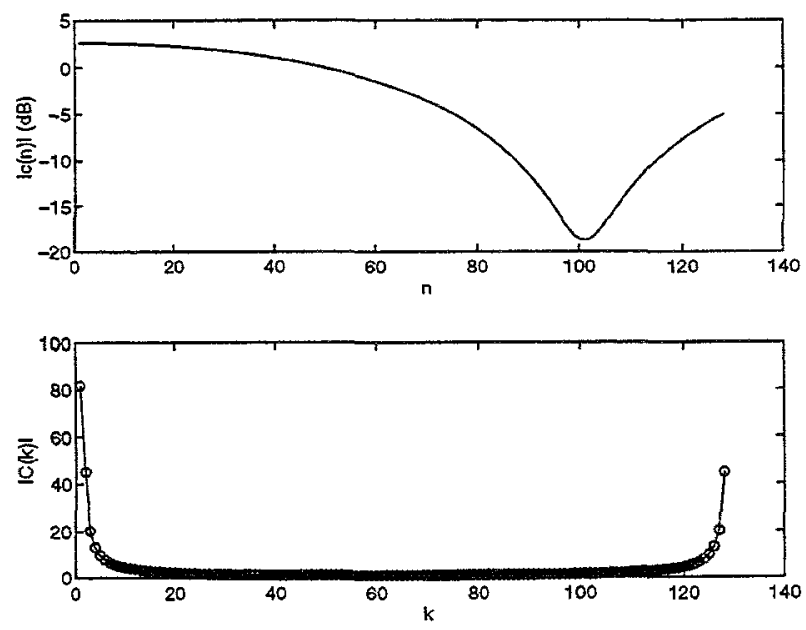

Fig.4. Typical realization of the Rayleigh fading channel $c(n)$ and its DFT $C(k)$.

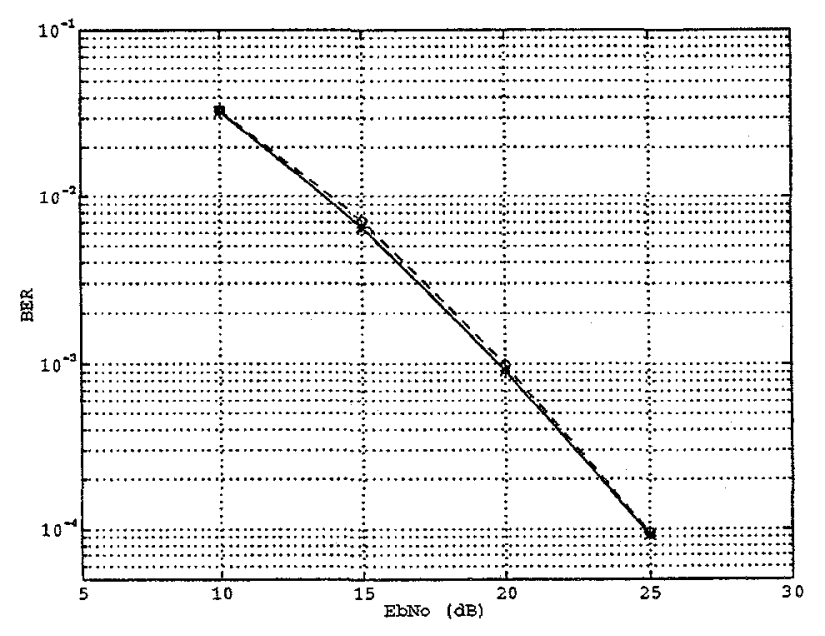

Fig.5. Performance of DFT (solid line) and WHT (dashed line) of length $N=128$. 